\title{
How long do patients with chronic disease expect to live? A systematic review of the literature
}

\author{
Authors: Barnaby Hole and Joseph Salem
}

\section{Aim}

To explore current understanding of self-estimated life expectancy amongst patients with non-cancer life-limiting disease.

\section{Methods}

A systematic search of the literature was performed of Medline, Embase, PsychINFO and the Cochrane Library from 1985 to 2015. Search terms relating to 'life expectancy' and 'selfestimation' were combined. Literature was included where adults with heart failure, chronic kidney disease, COPD or cirrhosis were asked to estimate their own life expectancy. Papers reporting only patients with malignant and infective disease, including HIV/AIDS, were excluded. 1,931 titles were searched independently by two reviewers. 39 papers were read, five of which met criteria for inclusion in the review.

\section{Results}

Evidence was rated as being of moderate quality in four, and of low quality in one, of the included papers. The best evidence was available for patients with heart failure and end-stage renal failure receiving haemodialysis. In outpatients with heart failure, self-estimated life expectancy was on average significantly greater than that predicted by a validated model. $66 \%$ of patients overestimated their survival, compared with the model, by $30 \%$ or more. The median overestimate was $40 \%$. Outpatients receiving haemodialysis were significantly more optimistic about their prognosis than their nephrologists, and when compared with prospective survival. Only $6 \%$ of patients thought they had a less than $50 \%$ chance of being alive at 5 years, whereas actual survival was only $56 \%$ by 2 years. Data available for patients with COPD were more limited and of insufficient quality to draw firm conclusions. No data on cirrhosis were found.

\section{Conclusions}

There is a paucity of published evidence examining selfestimated life expectancy among patients with non-cancer life-limiting disease. The available evidence showed that patients with heart and renal failure can make unrealistically optimistic predictions. The literature comes largely from single centre cohorts and is of moderate to low quality, and data from diseases other than heart and kidney failure are extremely limited. Clinicians should be aware that patients with chronic disease may have unrealistically optimistic expectations of their prognosis, and this may affect their health behaviour. 